\title{
A new landscape of civil engineering profession
}

\section{Opinion}

Civil and structural engineers have contributed enormously to the quality of life we enjoy today. The opportunities for them in the future are expected to be even greater if the profession can adapt to the changing needs. The new challenges in the future include, among others, deteriorating infrastructure and many pressing environmental issues. New physical infrastructure, including housing, water resources, and transportation systems must be properly designed to support the rapidly growing population, without imposing more environmental impacts like in the past.

In the past, changes in the civil engineering profession have always followed changes in technology and the society. Today's landscape of the profession is totally different from 30-40 years ago during which there has been a major change in technology and the new expectation from the society. Four major issues are identified here for the entire civil engineering community to consider in order to play meaningful roles in this fast changing world: (1) the incursion of new technologies and tools, synthesized from the existing knowledge; (2) the more holistic approach of engineering practice; (3) the regional and global integration of engineering service economy; and (4) the adaptation of engineering education.

New technologies have enabled civil engineers to practice with much higher degree of precision, certainty, and uniqueness. The speed and power of desktop computers and software enable engineers across the globe to compete and work as a team more equally. The increased computing capabilities in modeling, analysis and design have made the tedious and routine activities of traditional engineers obsolete, thus freeing them for more creative tasks. The global networking with broadband communications allows real-time collaboration between engineering design offices worldwide, thus redefining our perceptions of connectedness, location, and access. In the real world, experience has shown that social, environmental, economic, cultural, and ethical aspects of a project are often the deciding factors for its success, more than its technical aspect. Since the beginning of the twenty-first century, civil engineers have realized that they must embark to a more holistic approach to engineering. This requires a major paradigm shift from exploitation of nature to co-exist with nature. This requires an awareness of ecosystems, and the protection and restoration of natural capital. Civil engineers must also be trained in a "real-world" environment in which they will have to interact with other professionals from both technical and nontechnical disciplines. Clearly, they must be able to complement their technical capabilities

\author{
Volume 2 Issue 5 - 2017
}

\author{
Worsak Kanok Nukulchai \\ Department of Civil Engineeirng, Asian Institute of Technology, \\ Thailand
}

Correspondence: Worsak Kanok Nukulchai, Department of Civil Engineeirng, Asian Institute of Technology, Thailand, Emailworsak@gmail.com

Received: May 16, 2017 | Published: May 17, 2017

with a broad understanding of the so-called soft and worldly subjects. In the new world order, the world's economy has become closely linked, with changes sparked by technology. Civil engineers must be able to appreciate the diverse cultures in response to the needs of the multiple niches in a global market. These changes of professional landscape prompt the need to redesign educational curricula and programs to meet new challenges in the society and to provide the workforce required to integrate into the global economy. To adapt to all these changes, engineering curricula must be objectively designed and focused into 2 distinctive product lines, one to produce "builders of technology" and the other "users of technology". The idea is that knowledge cannot sell, but technology can. The first product line requires students to be trained on research and creativity, so they can have the skills to package knowledge into new technologies. For the second product line, the focus should be to educate students to be more technological proficient and broadly sophisticated in order to support economic growth and environmental preservation. In addition, engineering students must be prepared to enter the futuristic real world. In the next 20 years, this new breed of civil engineers will be working with diverse teams of engineers and non-engineers to formulate solutions to yet unknown problems. They must be equipped to be ready for emerging technologies in order to address more complex, larger scale multidisciplinary problems to meet the future needs of mankind.

\section{Acknowledgements}

None.

\section{Conflict of interest}

The author declares no conflict of interest. 